Editorial

\title{
Special Issue "Nanogrids, Microgrids, and the Internet of Things (IoT): Towards the Digital Energy Network"
}

\author{
Antonio Moreno-Munoz \\ Department of Electronics and Computer Engineering, University of Cordoba, E-14071 Cordoba, Spain; \\ el1momua@uco.es
}

Received: 16 September 2019; Accepted: 10 October 2019; Published: 14 October 2019

\section{Introduction}

I started hearing a lot about energy digitization a little over a year ago, talking to my colleagues during conferences and meetings. People did not agree with what they meant by "digitization". In my opinion, the power grid has long since ceased to be analog. The smart grid has always essentially been digital. So why this term, and why now? Is it just one of those buzzwords that are used for marketing purposes and does not really mean much at all? Once the word "smart" was exhausted, the technological gurus had to find another one that groups enough novelties and was broad as well. May not has been the right choice, but here we are, now with the need to give content or highlight some nuances that were no longer present in the pioneering smart grid concept.

A good approximation is the one we have used in the European Technology and Innovation Platform for Smart Networks for the Energy Transition Working Group on Digital Energy (ETIP SNET WG4). According to our position paper [1], digitization consists of "using digital technologies in order to change business models and provide new revenue streams and value-producing opportunities". This means that the Internet of Things, machine (M2M) communications, artificial intelligence, machine learning, digital twin, and other developments must find their use in the energy system. Indeed, the digitization of the energy system is not a recent development. Driven by new regulations, new market structures, and new energy resources, the smart grid has in recent decades been the trigger for profound changes in the way electricity is generated, distributed, managed and consumed. The smart grid has raised the traditional power grid by using two-way electricity and information flows to create an advanced, automated power supply network. Seen with insight, it could be said that smart grids have been the flagship application of digital technology in the energy sector. However, so far, the focus has been on the operation of the infrastructure. In the future energy systems, smart meters and appliances will expand the demand-response potential, enabling new relationships between the end-user and the energy system, and where service will be key.

In today's technological landscape, we can access feasible data and knowledge that has so far been merely inconceivable. This special issue aims to address this landscape towards which, the smart grid is progressing due to the advent of all these pervasive technologies. It will be the advanced exploitation of the massive data generated from internet of things (IoT) sensors that will become the main driver in evolving the concept of smart grids towards a digital energy network paradigm, focused on service. Furthermore, collective intelligence will improve the processes of decision making and empower citizens.

This issue includes outstanding cases of the use of these technologies and their advantageous energy system application at different levels, such as generation, distribution, or consumption. 


\section{A Short Review of the Contributions in This Issue}

The paper "A Novel Direct Load Control Testbed for Smart Appliances" [2] proposes a novel Direct Load Control (DLC) testbed, aiming to conveniently support the research community, as well as analyzing and comparing their designs in a laboratory environment. Based on the LabVIEW computing platform, this original testbed enables access to knowledge of major components such as online weather forecasting information, distributed energy resources (e.g., energy storage, solar photovoltaic), dynamic electricity tariff from utilities and demand response (DR) providers, together with different mathematical optimization features given by a General Algebraic Modelling System (GAMS). This intercommunication is possible thanks to the different applications' programming interfaces (API) incorporated into the system and to intermediate agents specially developed for this case. Different basic case studies have been presented to envision the possibilities of this system in the future and more complex scenarios, to actively support the DLC strategies. These measures will offer enough flexibility to minimize the impact on user comfort combined with support for multiple DR programs. Thus, given the successful results, this platform can lead towards a solution that has more efficient use of energy in the residential environment.

The paper "An Alternative Internet-of-Things Solution Based on LoRa for PV Power Plants: Data Monitoring and Management" [3] proposes a wireless low-cost solution based on long-range (LoRa) technology able to communicate with remote photovoltaic (PV) power plants, covering long distances with minimum power consumption and maintenance. This solution includes a low-cost open-source technology at the sensor layer and a low-power wireless area network (LPWAN) at the communication layer, combining the advantages of long-range coverage and low power demand. Moreover, it offers an extensive monitoring system to exchange data in an Internet-of-Things (IoT) environment. A detailed description of the proposed system at the PV module level of integration is also included in the paper, as well as detailed information regarding LPWAN application to the PV power plant monitoring problem. In order to assess the suitability of the proposed solution, results collected in real PV installations connected to the grid are also included and discussed.

The paper "Advantages of Minimizing Energy Exchange Instead of Energy Cost in Prosumer Microgrids" [4] two novel approaches are proposed: Firstly, a different objective function, relative to the mismatch between generated and demanded power is tested and compared to a classical objective function based on energy price, by means of a genetic algorithm method; secondly, this optimization procedure is applied to batteries' coordinated scheduling of all the prosumers composing a community, instead of single one, which better matches the microgrid concept. These approaches are tested on a microgrid with two household prosumers, in the context of Spanish regulation for self-consumption. Results show noticeably better performance of mismatch objective functions and coordinated scheduling, in terms of self-consumption and self-sufficiency rates, power and energy interchanges with the main grid, battery degradation, and even economic benefits.

In the paper "An Analysis of Voltage Quality in a Nanogrid during Islanded Operation" [5] voltage quality data has been collected in a single house nanogrid during 48 weeks of islanded operation and 54 weeks of grid-connected operation. The voltage quality data contains the voltage total harmonic distortion (THD), odd harmonics three to 11 and 15, even harmonics four to eight, voltage unbalance, short-term flicker severity (Pst) and long-term flicker severity (Plt) values, and voltage variations at timescales below $10 \mathrm{~min}$. A comparison between islanded and grid-connected operation values was made, where some of the parameters were compared to relevant grid-standard limits. It is shown that some parameters exceed the defined limits in the grid-standards during islanded operation. It was also found that the islanded operation has two modes of operation, one in which higher values of the short circuit impedance, individual harmonic impedance, harmonic voltage distortion, and voltage unbalance were reached.

In the paper "An Analysis of Frequency Variations and its Implications on Connected Equipment for a Nanogrid during Islanded Operation" [6] frequency, voltage and reliability data have been collected in a nanogrid for 48 weeks during islanded operation. Frequency values from the 48 -week measurements 
were analyzed and compared to relevant limits. During 19.5\% of the 48 weeks, the nanogrid had curtailed the production due to insufficient consumption in islanded operation. The curtailment of production was also the main cause of the frequency variations above the limits. When the microgrid operated on stored battery power, the frequency variations were less than in the Swedish national grid. Of all the interruptions that occurred in the nanogrid, $39.4 \%$ are also indirectly caused by the curtailment of solar production

A smart inverter should offer some features such as plug and play, self-awareness, adaptability, autonomy, and cooperativeness. These features are introduced and comprehensively explained in the paper "Smart Inverters for Microgrid Applications: A Review" [7]. In order to achieve higher functionality, efficiency and reliability, in addition to improving the control algorithms it is beneficial to equip the inverters with "smart" features. One interpretation of "smartness" refers to minimizing the requirement of communication and therefore switching from centralized to decentralized control. At the same time, being equipped with efficient and state of the art communication protocols also indicates "smartness" since the requirement of communication cannot be completely omitted. One contribution discussed here is the possibility of achieving long-range wireless communication between inverters to empower various control schemes. Although current efforts aim to modify and improve power converters in a way that they can operate communication free, if a suitable and functional communication protocol is available, it will improve the accuracy, speed, and robustness of them.

Conflicts of Interest: The authors declare no conflict of interest.

\section{References}

1. ETIP SNET WG4. Digitalization of the Energy System and Customer Participation. 2017. Available online: https://www.etip-snet.eu/wp-content/uploads/2018/11/ETIP-SNET-Position-Paper-on-Digitalisationshort-for-web.pdf (accessed on 13 October 2019).

2. Garrido-Zafra, J.; Moreno-Munoz, A.; Gil-de-Castro, A.; Palacios-Garcia, E.J.; Moreno-Moreno, C.D.; Morales-Leal, T. A Novel Direct Load Control Testbed for Smart Appliances. Energies 2019, 12, 3336. [CrossRef]

3. Paredes-Parra, J.M.; García-Sánchez, A.J.; Mateo-Aroca, A.; Molina-Garcia, A. An Alternative Internet-of-Things Solution Based on LoRa for PV Power Plants: Data Monitoring and Management. Energies 2019, 12, 881. [CrossRef]

4. González-Romera, E.; Ruiz-Cortés, M.; Milanés-Montero, M.I.; Barrero-González, F.; Romero-Cadaval, E.; Lopes, R.; Martins, J. Advantages of Minimizing Energy Exchange Instead of Energy Cost in Prosumer Microgrids. Energies 2019, 12, 719. [CrossRef]

5. Nömm, J.; Rönnberg, S.; Bollen, M. An Analysis of Voltage Quality in a Nanogrid during Islanded Operation. Energies 2019, 12, 614. [CrossRef]

6. Nömm, J.; Rönnberg, S.; Bollen, M. An Analysis of Frequency Variations and its Implications on Connected Equipment for a Nanogrid during Islanded Operation. Energies 2018, 11, 2456. [CrossRef]

7. Arbab-Zavar, B.; Palacios-Garcia, E.; Vasquez, J.; Guerrero, J. Smart Inverters for Microgrid Applications: A Review. Energies 2019, 12, 840. [CrossRef]

(C) 2019 by the author. Licensee MDPI, Basel, Switzerland. This article is an open access article distributed under the terms and conditions of the Creative Commons Attribution (CC BY) license (http://creativecommons.org/licenses/by/4.0/). 